\title{
Фазообразование и кинетика кристаллизации аморфных пленок Culn $\mathbf{S}_{8}$
}

\author{
(С) Г.Е. Дашдамирова ${ }^{1}$, Э.Б. Аскеров ${ }^{2,3}$, Д.И. Исмаилов ${ }^{1}$ \\ ${ }^{1}$ Институт физики Национальной академии наук, \\ AZ1143 Баку, Азербайджан \\ ${ }^{2}$ Объединенный институт ядерных исследований, \\ 141980 Дубна, Россия \\ ${ }^{3}$ Национальный центр ядерных исследований, \\ 370143 Баку, Азербайджан \\ E-mail: elmar.asgerov@gmail.com
}

Поступила в Редакцию 17 ноября 2021 г. В окончательной редакции 25 ноября 2021 г. Принята к публикации 25 ноября 2021 г.

Исследованы процессы взаимодействия и образования фаз в системе $\mathrm{Cu}_{2} \mathrm{~S}-\mathrm{In}_{2} \mathrm{~S}_{3}$, полученных последовательным и одновременным осаждением двойных соединений составов $\mathrm{Cu}_{2} \mathrm{~S}$ и $\operatorname{In}_{2} \mathrm{~S}_{3}$ в вакууме. Методом кинематической электронографии установлены кинетические параметры кристаллизации аморфных пленок состава $\mathrm{CuIn}_{5} \mathrm{~S}_{8}$, образующихся в результате вакуумной конденсации указанных выше двойных соединений как в обычных условиях, так и в условиях воздействия внешнего электрического поля.

Ключевые слова: кинематическая электронография, наноразмерные пленки, плоскость конденсации.

DOI: $10.21883 /$ FTP.2022.03.52114.9773

\section{1. Введение}

Полупроводниковое тройное соединение $\mathrm{CuIn}_{5} \mathrm{~S}_{8}$ принадлежит к полупроводникам типа $\mathrm{M} \mathrm{B}_{5}^{\mathrm{III}} \mathrm{C}_{8}^{\mathrm{VI}}(\mathrm{M}-\mathrm{Cu}$, $\mathrm{Fe}, \mathrm{Mn} ; \mathrm{B}^{\mathrm{III}}$ - In, Ga, Al; $\left.\mathrm{C}^{\mathrm{VI}}-\mathrm{S}, \mathrm{Se}, \mathrm{Te}\right) . \mathrm{CuIn}_{5} \mathrm{~S}_{8}$ обладает упорядоченными дефектами с концентрацией $\sim 25 \%$. В работе [1] указана перспективность данного соединения для создания высокоэффективных солнечных элементов - широкополосных преобразователей естественного излучения, поскольку при $T=300 \mathrm{~K}$ его фоточувствительность изменяется в диапазоне значений $1.2-3.5$ эВ. Оптимальный для преобразования солнечной энергии диапазон значений составляет $1.2-1.4$ эВ. Согласно [2-4], соединение $\mathrm{CuIn}_{5} \mathrm{~S}_{8}$ наряду с эффективными оптическими излучателями может быть использовано и в качестве материала для создания инфракрасных детекторов и гетеропереходов различных типов. В системе $\mathrm{Cu}-\mathrm{S}$ существуют три устойчивые самостоятельные фазы: $\mathrm{Cu}_{9} \mathrm{~S}_{5}$ (дигенит), $\mathrm{CuS}$ (ковелин) и $\mathrm{Cu}_{2} \mathrm{~S}$ (халькозин) плотностью $\rho=5.8 \mathrm{r} / \mathrm{cm}^{3}$. Халькозин обладает двумя модификациями: низкотемпературной и высокотемпературной [5]. По данным работы [5], низкотемпературный $\mathrm{Cu}_{2} \mathrm{~S}$ относится к пространственной группе симметрии (ПГС) $A b 2 m\left(C_{2 V}^{15}\right)$ и обладает ромбической ячейкой с $a=11.90, b=27.28, c=13.41 \AA$. Постоянные гексагональной решетки с ПГС $C_{6} / m m c$ высокотемпературного халькозина, существующего при $>378 \mathrm{~K}$, составляют: $a=3.89, c=6.68 \AA$.

Термодинамическим анализом системы In-S в [5] было обнаружено четыре сульфида: $\mathrm{InS}_{,} \mathrm{In}_{5} \mathrm{~S}_{6}, \mathrm{In}_{3} \mathrm{~S}_{4}$ и $\mathrm{In}_{2} \mathrm{~S}_{3}$, плавящихся конгруэнтно при температуре $1363 \mathrm{~K}$. Соединение $\operatorname{In}_{2} \mathrm{~S}_{3}$, так же, как и $\mathrm{Cu}_{2} \mathrm{~S}$, является диморфным и обладает низкотемпературной и высокотемпе- ратурной $\alpha$ и $\beta$ фазами соответственно. Выше $573 \mathrm{~K}$ $\alpha$-модификация необратимо переходит в высокотемпературную $\beta$-модификацию со структурой типа шпинели. Кристаллографические постоянные $\alpha$ - $\operatorname{In}_{2} \mathrm{~S}_{3}$, согласно данным работы [5], имеют гранецентрированную кубическую решетку с $a=5.37 \AA$, ПГС $F d 3 m-O_{h}^{7}$, рентгено-

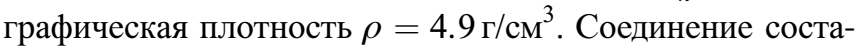
ва $\mathrm{CuIn}_{5} \mathrm{~S}_{8}$ кристаллизуется в кубической сингонии с периодами элементарной ячейки $a=10.685 \AA$ [2] и описывается как усложненная структура шпинеля с ПГС кристаллов $F d 3 m$. Приведенные в [1-5] данные о фазовых составах и структурах относятся к объемным образцам.

Настоящая работа посвящена электронографическим исследованиям взаимодействия тонких пленок двойных соединений $\mathrm{Cu}_{2} \mathrm{~S}$ и $\mathrm{In}_{2} \mathrm{~S}_{3}$, образования тройного соединения $\mathrm{CuIn}_{5} \mathrm{~S}_{8}$ в тонкопленочном состоянии и механизма кинетики кристаллизации аморфных пленок $\mathrm{CuIn}_{5} \mathrm{~S}_{8}$, которые не могут быть заменены другими исследованиями и являются важными задачами современного материаловедения - получения наноразмерных полупроводниковых материалов с принципиально новыми физическими, химическими и другими свойствами. Процессы фазообразования и структурные изменения исследованы на электронографе ЭМР-102. Фазовые превращения, вызванные внешними воздействиями, изучены методом кинематической электронографии, применение которого приводит к качественному переходу от исследований массивных образцов к исследованиям тончайших слоев химических соединений и их твердых растворов в пленочном исполнении. Методом кинематической электронографии, являющимся одним из методов дифракции электронов высоких энергий (ДЭВЭ), изучая 
температурно-временну́ю зависимость кристаллизации аморфных пленок, определяются стационарные значения энергий активации образования центров кристаллизации и дальнейшего их роста. Исследуемые тонкие пленки, конденсированные в обычных условиях и в условиях воздействия внешнего электрического поля напряженностью $500 \mathrm{~B} \cdot \mathrm{cm}^{-1}$, были получены в вакуумном испарителе в сопряжении с универсальным источником постоянного тока УИП-1. Давление остаточных газов в вакуумном испарителе было $\leq 10^{-5}$ Па

\section{2. Экспериментальные методы}

С целью получения наноразмерных пленок состава $\mathrm{CuIn}_{5} \mathrm{~S}_{8}$ и дальнейшего их исследования методом ДЭВЭ из двух независимых источников производили испарение навесок двойных соединений $\mathrm{Cu}_{2} \mathrm{~S}$ и $\mathrm{In}_{2} \mathrm{~S}_{3}$ в соотношении 1:5. Навески испарялись из конически навитых вольфрамовых спиралей, мощности которых позволяли производить испарение полностью и с большой скоростью, исключая разложение и обеспечивая идентичность составов исходных фаз и полученных при осаждении тонких пленок. При последовательной и одновременной конденсации навески $\mathrm{Cu}_{2} \mathrm{~S}$ и $\mathrm{In}_{2} \mathrm{~S}_{3}$ ( 2.5 и 12 мг соответственно) помещались в источники испарения, удаленные друг от друга на 120 мм. Источники находились на высоте 70 мм от плоскости подложек, которые обезгаживались в вакууме при температуре $523 \mathrm{~K}$ в течение 2 ч. Подложками служили свежие сколы монокристаллов $\mathrm{NCl}$ и $\mathrm{KCl}$ микронной толщины. Известно, что в процессе фазообразования порядок напыления компонентов играет важную роль. Были проведены две серии опытов. В одном случае $\mathrm{Cu}_{2} \mathrm{~S}$ испарялся на слой $\operatorname{In}_{2} \mathrm{~S}_{3}$, предварительно осажденный на подложки, находящиеся при комнатной температуре, а в другом - $\mathrm{In}_{2} \mathrm{~S}_{3}$ на $\mathrm{Cu}_{2} \mathrm{~S}$. Испарение проводилось со скоростью $\sim 20-25 \AA /$ c.

Так как $\mathrm{Cu}_{2} \mathrm{~S}$ и $\mathrm{In}_{2} \mathrm{~S}_{3}$ сильно отличаются друг от друга характером испарения, то одновременное осаждение паров данных соединений потребовало предварительного изучения режима испарения отдельно каждого из них, что было необходимо для их синхронного испарения. Вещества испарялись из танталовых лодочек. Предварительные опыты показали, что $\mathrm{Cu}_{2} \mathrm{~S}$ очень быстро и легко испаряется уже при очень малом токе накала „лодочки“. В противоположность ему $\operatorname{In}_{2} \mathrm{~S}_{3}$ испаряется весьма своеобразно. Уже при незначительном нагреве лодочки $\operatorname{In}_{2} \mathrm{~S}_{3}$ плавятся. Чуть больший накал приводит к растеканию $\mathrm{In}_{2} \mathrm{~S}_{3}$. Только при белом калении лодочки $\operatorname{In}_{2} \mathrm{~S}_{3}$ интенсивно испаряются. Опираясь на эти факты, мы и проводили одновременное испарение данных веществ. Сначала давался накал на лодочку $\mathrm{In}_{2} \mathrm{~S}_{3}$, который плавился и начинал смачивать стенки лодочки. В этот момент включался накал лодочки $\mathrm{Cu}_{2} \mathrm{~S} . \mathrm{Cu}_{2} \mathrm{~S}$ почти сразу начинал испаряться, и в это же время лодочка $\operatorname{In}_{2} \mathrm{~S}_{3}$ доводилась до белого каления. Весь процесс длился $\sim 7-10$ с.
Для предотвращения окисления и реиспарения пленок в процессе последующей термообработки пленки помещались в углеродные капсулы, т. е. применяли разновидность метода капсулирования исследуемых пленок, впервые предложенного в работах [6,7]. Анализ соединений, заключенных в углеродной капсуле, с одной стороны, затрудняет сам процесс эксперимента, но с другой дает возможность получить более объективный результат: методика капсулирования пленок требует особенной тщательности, позволяющей, однако, минимизировать эффекты с поглощением образцами влаги из атмосферного воздуха и при термообработке образцов. Толщина исследуемых пленок, с учетом углеродных, не превышала $500 \AA$, система сплавов, полученная таким образом, с плавно изменяющимся составом содержит в себе от 0 до $100 \% \mathrm{Cu}_{2} \mathrm{~S}$, от 100 до $0 \% \mathrm{In}_{2} \mathrm{~S}_{3}$ по краям плоскости конденсации.

Обычная дифракционная картина поликристаллического вещества представляет собой серию концентрических колец различной интенсивности. При исследовании кинетики фазовых переходов в тонких слоях на одну фотопластинку можно было бы снимать серию электронограмм с различной термообработкой, но эти снимки будут делаться через какой-то промежуток времени, в течение которого в исследуемом объекте могут произойти фазовые изменения. Поэтому возникает необходимость с параллельным нагревом пленок, непрерывно фиксировать дифракционную картину.

Для получения кинематических электронограмм, от дифракционной картины поликристаллического образца, с помощью экрана с узкой щелью выделялся участок дифракционного поля вдоль горизонтального диаметра. Электронограммы в этом случае выглядят как серия коротких дуг, близких к прямым линиям. При движении фотопластинки перед щелью на ней фиксируются параллельные линии, расположение и интенсивности которых меняются в зависимости от изменения структуры и состояния вещества. При ускоряющем напряжении 50 кВ постоянная электронографа составляла $2 L \lambda=70.5 \mathrm{MM} \cdot \AA$.

\section{3. Результаты и обсуждение}

Для получения пленок, полностью структурно сформированных и строго стехиометрических составов, при изучении процессов взаимодействия и образования фаз нами был использован метод, примененный в [6,7], позволяющий контролировать геометрически заданную толщину формирующихся пленок. Количественный состав вещества, распределение состава конденсата по координатам на единице поверхности плоскости конденсации определяли по формуле

$$
\mathbf{q}=\frac{Q}{4 \pi h^{2}} \frac{1}{(1+\lambda)^{3 / 2}} .
$$

Здесь $\mathbf{q}$ - количество вещества на единице поверхности коллектора, $Q$ - количество испаряемого вещества, 
$h$ - расстояние от источника испарения до любой точки плоскости распределения конденсата, коэффициент $\lambda=x / h$, где $x$ - расстояние от точки, находящейся непосредственно под испарителем, до какой-либо точки распределенного конденсата по всей площади коллектора. Толщина пленок вычислялась по формуле

$$
H=\frac{q}{\rho},
$$

где $\rho-$ плотность вещества в г/ $\mathrm{cm}^{3}$.

При одновременной и послойной конденсации $\mathrm{Cu}_{2} \mathrm{~S}$ и $\mathrm{In}_{2} \mathrm{~S}_{3}$, независимо от последовательности напыления нами было получено 30 образцов, которые все были электронографированы. Расчет электронограмм от объектов, полученных методом одновременного испарения, показал, что распределение фаз на плоскости конденсации не отличается от случая раздельного их испарения, т.е. по реакциям взаимодействия закономерность образования фаз не меняется - интервал составов и протяженность образующихся фаз на подложках остаются неизменными. Электронографическое исследование пленок, образующихся при осаждении паров $\mathrm{Cu}_{2} \mathrm{~S}$ и $\mathrm{In}_{2} \mathrm{~S}_{3}$ на подложках, находящихся при комнатной температуре, показало, что на плоскости конденсации, начиная от подложек, расположенных непосредственно под источником $\mathrm{In}_{2} \mathrm{~S}_{3}$ в сторону испарителя $\mathrm{Cu}_{2} \mathrm{~S}$, в широкой области образуются аморфные пленки. Наблюдается лишь одна кристаллическая фаза, формирующаяся на подложках, находящихся под испарителем $\mathrm{Cu}_{2} \mathrm{~S}$. Значения $\rho=4 \pi \sin \theta / \lambda$, соответствующие диффузным ореолам, наблюдаемым на электронограммах, полученных от аморфных пленок, образующихся на подложках, находящихся под источником $\mathrm{In}_{2} \mathrm{~S}_{3}$, составляют 2.211, $3.388,5.365 \AA^{-1}$, кристаллизация которых приводит к образованию кубической структуры с параметрами, приведенными в работе [5].

Термообработка аморфных пленок с $\rho$ равными 1.892 , $3.094,4.331 \AA^{-1}$, расположенных по средине плоскости конденсации, позволила выявить поликристаллический $\mathrm{CuIn}_{5} \mathrm{~S}_{8}$ с периодами, указанными в работе [2]. Схема распределения фаз, построенная на основе электронографического анализа пленок, полученных на подложках монокристаллических $\mathrm{NCl}, \mathrm{KCl}$, расположенных друг от друга на расстоянии $3 \mathrm{MM}$, при общей длине плоскости конденсации 120 мм, показала, что во всех случаях независимо от способа получения выявляются резкие границы раздела между аморфными и формирующимися в кристаллическом состоянии пленками.

Кинематические электронограммы от аморфных пленок, кристаллизующихся в кубической сингонии $\mathrm{CuIn}_{5} \mathrm{~S}_{8}$, в которых прослеживаются области существования обеих фаз (аморфной и кристаллической) и изменения интенсивностей дифракционных линий растущей кристаллической фазы, соответствующие различным моментам времени, получены при температурах 403, 418, $433 \mathrm{~K}$. На рис. 1 представлена кинематическая электронограмма, полученная при $418 \mathrm{~K}$.

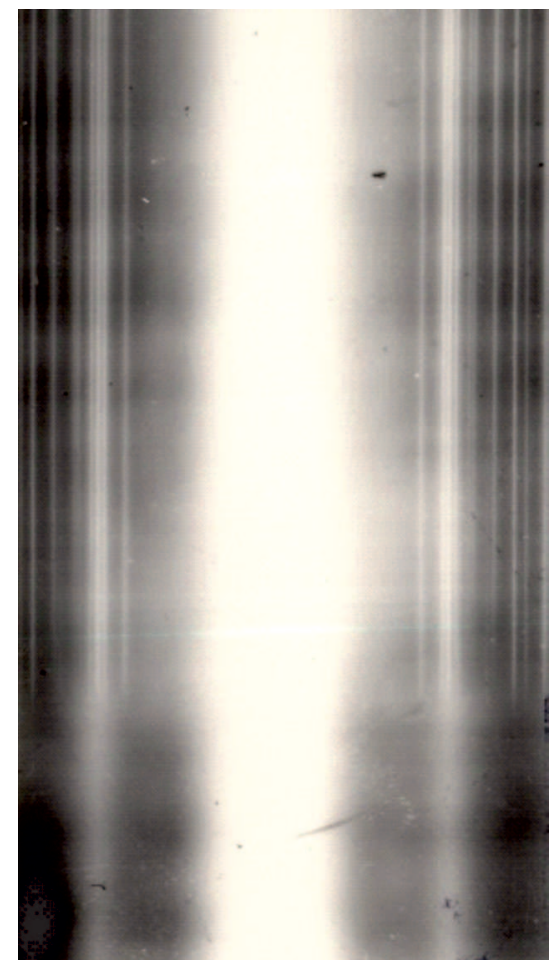

Рис. 1. Кинематическая электронограмма от аморфного $\mathrm{CuIn}_{5} \mathrm{~S}_{8}$, претерпевающего фазовое превращение при $418 \mathrm{~K}$.

Пороговая температура для аморфного $\mathrm{CuIn}_{5} \mathrm{~S}_{8}$ coставляла $458 \mathrm{~K}$, при которой происходила моментальная кристаллизация аморфной пленки, что не позволяло проследить всю динамику процесса фазового перехода. Дифракционные линии на кинематической электронограмме от поликристалла индицируются на основе периодов со значениями, приведенными в работе [2]. На кинематической электронограмме усиление со временем интенсивностей дифракционных линий от кристаллической фазы при кристаллизации обусловлено увеличением объема поликристаллической фазы, облучаемого электронным пучком, т.е. изменение интенсивностей дифракционных линий одной фазы связано с изменением количества этой фазы в облучаемом объеме, так как общее количество вещества остается неизменным. Так как на кинематической электронограмме фиксируется небольшой участок от дифракционной картины (ДК), то локальная интенсивность дебаевского кольца, согласно данным работы [8], выражается формулой

$$
I_{h k l}=I_{0} \lambda\left[\frac{\Phi_{h k l}}{\Omega}\right]^{2} V \frac{d_{h k l} \Delta P}{4 \pi L \lambda}
$$

Здесь $I_{0}-$ интенсивность первичного пучка излучения электронов, $\lambda$ - длина электронной волны, $\Phi-$ структурная амплитуда дифракционного рефлекса, которая в кинематическом приближении вычисляется из атомных факторов рассеяния электронов, $\Omega$ - объем элементарной ячейки, $V$ - облучаемый объем поли- 


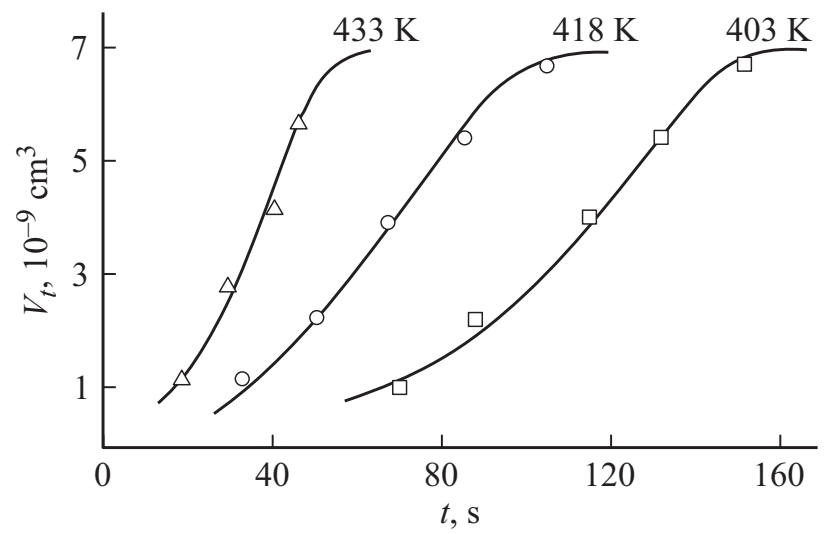

Рис. 2. Кинетические кривые кристаллизации аморфного $\mathrm{CuIn}_{5} \mathrm{~S}_{8}$.

кристаллического препарата, $d_{h k l}$ и $\Delta-$ межплоскостное расстояние и малый участок дебаевского кольца соответственно, $P$ - фактор повторяемости усиления дифракционного отражения, $L \lambda$ - постоянная прибора, зависящая от приложенного напряжения, ускоряющего электроны.

При кинематических съемках значения величин, входящих в правую часть формулы (3), остаются постоянными, кроме $V$. Интенсивность дифракционного отражения с индексами $h k l$ при условии постоянства $I_{0}$ и $L \lambda$, согласно (8), пропорциональна облучаемому объему поликристаллического вещества, т.е. $I_{h k l} \sim V$. Следовательно, по кинематическим электронограммам, определив изменение интенсивности дифракционных линий, можно найти изменения количества вещества, претерпевающего фазовое превращение. Полностью закристаллизованному объему $-S \cdot h$ нами сопоставлялось соответствующее ему максимальное значение интенсивности, получаемое при насыщении, где $S$ - площадь сечения электронного пучка, равная $\sim 2.8 \cdot 10^{-3} \mathrm{~cm}^{2}$, $h$ - толщина пленки, которая составляла $250 \AA$.

Микрофотометрированием различных участков кинематических электронограмм в зависимости от продолжительности времени термообработки пленок определялись интенсивности линий ДК, принимающие индексы (220), (320) и (402). Значения объема кристаллизующейся части $\mathrm{CuIn}_{5} \mathrm{~S}_{8}$, определенные по указанным дифракционным рефлексам, примерно одинаковы, предельные значения составляют:

$$
V=S \cdot h=8 \cdot 10^{3} \mathrm{~cm}^{2} \cdot 2.5 \cdot 10^{-6} \mathrm{~cm}=7 \cdot 10^{-9} \mathrm{~cm}^{3} .
$$

Полученное значение совпадает также с величиной, найденной из следующих рассуждений: так как радиус электронно-лучевого пучка составляет $0.3 \mathrm{Mм}$ $\left(S=2.8 \cdot 10^{-3} \mathrm{~cm}^{2}=\pi r^{2}, r=\sqrt{\left(28 \cdot 10^{-4}\right) / \pi}=3 \cdot 10^{-2} \mathrm{~cm}\right)$, то $\quad V=\pi r^{2} h=S \cdot h=\left(2.8 \cdot 10^{-3} \mathrm{~cm}^{2}\right) \cdot 2.5 \cdot 10^{-6} \mathrm{~cm}$ $=7 \cdot 10^{-9} \mathrm{~cm}^{3}$.

На рис. 2 представлена зависимость объема закристаллизовавшейся части $\mathrm{CuIn}_{5} \mathrm{~S}_{8}$ от времени отжига при температурах 403, 418, 433 K, т. е. кинетические кривые кристаллизации аморфного $\mathrm{CuIn}_{5} \mathrm{~S}_{8}$, кристаллизующиеся в кубической сингонии.

Аналитическое выражение, описывающее кинетические закономерности фазовых превращений, получено в работе [9] в виде уравнения

$$
V_{t}=V_{0}\left[1-\exp \left(-k t^{m}\right)\right] .
$$

Здесь $V_{t}$ - объем закристаллизовавшегося вещества к моменту $t, V_{0}$ - начальный объем, $k$ - константа скорости реакции, равная $\frac{1}{3} \pi v_{3} v_{p}^{3}$, где $v_{3}$ и $v_{p}-$ скорости образования кристаллических зародышей и дальнейшего их роста соответственно, которые описываются выражениями

$$
v_{3}=A_{1} \exp \left(-\frac{E_{3}}{R T}\right), \quad v_{p}=A_{2} \exp \left(-\frac{E_{p}}{R T}\right),
$$

где $A_{1}$ и $A_{2}-$ не зависящие от температуры некоторые постоянные, $E_{3}$ и $E_{p}$ - энергии активации зародышеобразования и роста соответственно, $R$ - универсальная газовая постоянная, $T$ - абсолютная температура. Величина $\mathrm{m}$ различна для возможных типов превращений и зависит от мерности роста кристалликов.

Для сравнения изотермических кривых с уравнением (4), позволяющим установить закономерности кинетики фазовых превращений, данное аналитическое уравнение может быть представлено в виде

$$
\ln \ln \frac{V_{0}}{V_{0}-V_{t}}=\ln k+m \ln t .
$$

На основе экспериментальных данных для указанных выше температур построены графики зависимости $\ln \ln \frac{V_{0}}{V_{0}-V_{t}}$ от $\ln t$ (рис. 3), в которых экспериментально найденные точки для всех трех температур укладываются на прямые линии, указывающие на то, что экспериментальные изотермы хорошо описываются выражением, установленным Аврами-Колмогоровым. Из наклона

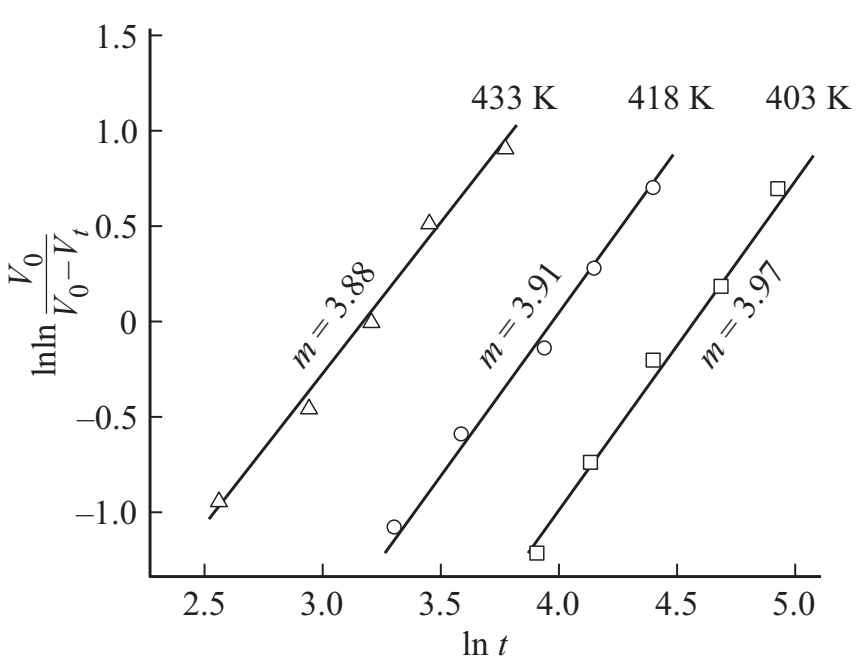

Рис. 3. Зависимость $\ln \ln \frac{V_{0}}{V_{0}-V_{t}}$ от $\ln t$ для кристаллизации аморфного $\mathrm{CuIn}_{5} \mathrm{~S}_{8}$. 
прямых к оси абсцисс, приведенных на рис. 3, установленные значения для показателя степени $t$ оказались равными $\sim 4(m=3.88$ для $433 \mathrm{~K}, m=3.91$ для $418 \mathrm{~K}$, $m=3.97$ для $403 \mathrm{~K})$.

Таким образом, сопоставление экспериментальных изотерм с аналитическим выражением показало, что лучшее совпадение имеет место при $m \approx 4$. Полученное для $m$ данное значение показывает, что в случае кристаллизации аморфных пленок $\mathrm{CuIn}_{5} \mathrm{~S}_{8}$ имеет место трехмерный рост кристалликов. Согласно (5), пересечение прямых зависимости $\ln \ln \frac{V_{0}}{V_{0}-V_{t}}$ от $\ln t$ с осью ординат определяют значения $\ln k$. Значения $\ln k$, полученные нами для указанных выше температур, оказались следующими: при $403 \mathrm{~K} \ln k=-25.4$, при $418 \mathrm{~K} \ln k=-19.7$, при $433 \mathrm{~K} \ln k=-15.5$. По наклону прямой зависимости $\ln k$ от обратной температуры определена общая энергия активации кристаллизации аморфного $\mathrm{CuIn}_{5} \mathrm{~S}_{8}$, состоящая из двух слагаемых: $E_{\text {sum }}=E_{3}+E_{p}$. Ее значение равно 75.9 ккал/моль. Скорость образования центров кристаллизации адекватно описывается обратным значением инкубационного времени $1 / \tau_{0}$, т. е. характеризуется как $E_{3} \sim 1 / \tau_{0}$. Энергия активации зародышеобразования, вычисленная по наклону прямой зависимости $\ln 1 / \tau_{0}$ от обратной температуры, оказалась равной 23.2 ккал/моль. Энергия активации роста кристалликов, установленная из соотношения $E_{p}=\left(E_{\mathrm{sum}}-E_{3}\right) / 3$, равна 17.6 ккал/моль.

Исследование кинетики кристаллизации аморфных пленок $\mathrm{CuIn}{ }_{5} \mathrm{~S}_{8}$, осажденных при воздействии внешнего электрического поля, проводилось также как для пленок, конденсированных в обычных условиях. Для кристаллизации тонких аморфных слоев $\mathrm{CuIn}_{5} \mathrm{~S}_{8}$ в этом случае при всех исследуемых температурах в интервале $403-433 \mathrm{~K}$ требуется больше времени для кристаллизации пленок, полученных вне поля. Суммарная энергия активации $\left(E_{\text {sum }}\right)$ процесса кристаллизации пленок, формирующихся в условиях воздействия электрического поля, величины энергий активации зародышеобразования $\left(E_{3}\right)$ и роста $\left(E_{p}\right)$ определялись аналогично случаю отсутствия поля и оказались равными 88.5, 26.7, 20.6 ккал/моль соответственно, они были больше соответствующих величин для пленок, полученных в обычных условиях: образующиеся под воздействием электрического поля аморфные слои $\mathrm{CuIn}_{5} \mathrm{~S}_{8}$ более устойчивы, чем в случае отсутствия поля.

\section{4. Заключение}

При одновременном и послойном осаждении $\mathrm{Cu}_{2} \mathrm{~S}$ и $\operatorname{In}_{2} \mathrm{~S}_{3}$, независимо от последовательности напыления, интервал составов и протяженности на плоскости конденсации образующихся фаз, оставаясь неизменными, реализуются аморфные фазы составов $\mathrm{In}_{2} \mathrm{~S}_{3}, \mathrm{CuIn}_{5} \mathrm{~S}_{8}$ и одна кристаллическая фаза состава $\mathrm{Cu}_{2} \mathrm{~S}$.

Кинетика кристаллизации аморфных пленок $\mathrm{CuIn}{ }_{5} \mathrm{~S}_{8}$, полученных осаждением молекулярных пучков $\mathrm{Cu}_{2} \mathrm{~S}$ и $\mathrm{In}_{2} \mathrm{~S}_{3}$ в обычных условиях и в условиях воздействия на них электрического поля напряженностью $500 \mathrm{~B} \cdot \mathrm{cm}^{1}$, подчиняется закономерностям, установленным Аврами-Колмогоровым, и описывается выражением $V_{t}=V_{0}\left[1-\exp \left(-k t^{m}\right)\right]$. В аморфных пленках при их кристаллизации происходит трехмерный рост кристалликов. Значения энергий активации зародышеобразования и роста кристалликов в пленках, полученных в условиях воздействия электрического поля, на $\sim 15 \%$ больше, чем соответствующие значения для пленок, полученных вне поля.

\section{Конфликт интересов}

Авторы заявляют, что у них нет конфликта интересов.

\section{Список литературы}

[1] И.В. Боднарь, М.А. Жафар. Докл. БГУИР, 5(107), 40 (2017).

[2] S. Kitamura, S. Endo, T. Irie. J. Phys. Chem. Solids, 8 (46), 881 (1985).

[3] Н.С. Орлова, И.В. Боднарь, Е.А. Кудрицкая. Неорг. матер., 8 (33), 932 (1997).

[4] И.В. Боднарь. ФТП, 32 (9), 1043 (1998).

[5] М. Хансен, К. Андерко. Структура двойных сплавов (М., Металлургиздат, 1962) т. 2, с. 1488.

[6] Э.Б. Аскеров, А.И. Мададзада, Д.И. Исмаилов, Р.Н. Мехтиева. ФТП, 48 (9), 1265 (2014).

[7] Э.Б. Аскеров, А.И. Мададзада, Д.И. Исмаилов, Р.Н. Мехтиева. ФТП, 48 (11), 1484 (2014).

[8] Б.К. Вайнштейн. Структурная электронография (М., Изд-во АН СССР, 1956) с. 313.

[9] M. Avrami. J. Chem. Phys., 8 (2), 212 (1940).

Редактор А.Н. Смирнов

\section{Phase formation and crystallization kinetics of amorphous Culn $\mathbf{n}_{5} \mathbf{S}_{8}$ films}

\author{
G.E. Dashdamirova ${ }^{1}$, E.B. Asgerov ${ }^{2,3}$, D.I. Ismailov ${ }^{1}$ \\ ${ }^{1}$ Institute of Physics, \\ National Academy of Sciences of Azerbaijan, \\ AZ1143 Baku, Azerbaijan \\ 2 Joint Institute for Nuclear Research, \\ 141980 Dubna, Russia \\ ${ }^{3}$ National Nuclear Research Centre, \\ 370143 Baku, Azerbaijan
}

\begin{abstract}
The processes of interaction and formation of phases in the $\mathrm{Cu}_{2} \mathrm{~S}-\mathrm{In}_{2} \mathrm{~S}_{3}$ system, obtained by sequential and simultaneous deposition of binary compounds of the $\mathrm{Cu}_{2} \mathrm{~S}$ and $\mathrm{In}_{2} \mathrm{~S}_{3}$ compositions in vacuum, have been investigated. Using the method of kinematic electron diffraction, the kinetic parameters of crystallization of amorphous films of the composition $\mathrm{CuIn}_{5} \mathrm{~S}_{8}$, formed as a result of vacuum condensation of the above binary compounds, both under normal conditions and under the influence of an external electric field, have been established.
\end{abstract}

